\title{
Towards a sustainable community database: taking advantage of the Road-to-Health cards to monitor and evaluate health interventions targeting under fives
}

\author{
D.O. SIMBA \\ Muhimbili University of Health and Allied Sciences, P. O. Box 65015, Dar es Salaam, Tanzania \\ E-mail address: dsimba@muhas.ac.tz; daudisimba@yahoo.com
}

\begin{abstract}
The Road-to-Health (RTH) card has served as a tool for monitoring nutrition and vaccination status individual child for several decades. The card has the potential to serve as a community database for research if kept by the caretaker for a considerable period. This study aimed to assess whether the magnitude of possession and retention of RTH cards is adequate to serve as a community database for monitoring and evaluating health interventions targeting under fives. This cross-sectional study was conducted among under fives in Korogwe town and its suburbs in Tanzania. Six wards and four villages were randomly selected and all under fives found were included. Using a structured questionnaire, demographic information was obtained from the parent/guardian of the child. Information was collected on the presence of RTH card from which the date of birth was recorded. A total of 4899 households were involved and information obtained for 6364 under fives. The overall card possession rate was 74.3\%. Possession of RTH cards was found to be highest among the last born under fives (78.3\%) than the third-from-last born under fives (45.1\%). Caretakers who were married and educated had higher card possession rate. In conclusion the possession of RTH cards was adequate to serve as a community database for monitoring health status and evaluating health interventions targeting the under fives. However, the low retention rate poses a limitation for the cards to serve as a permanent community database. This paper discusses some of the strategies to increase retention of the cards by caretakers.
\end{abstract}

Key words: Road-to-health card, community, database, child, health information, Tanzania

\section{Introduction}

Community-based Health Information System (CBHIS) has the advantage of being able to measure the outcome and impact of health interventions which is not possible with facility based health information systems in low income countries where a large proportion of the population do not utilise formal health care services (Kumar, 1993). In these countries, monitoring and evaluation of health interventions at community level is limited by the lack of a sustainable community based information system. In Tanzania, thereare several systems for data collection that are based at the community level such as, the village registration, child village weighing programme and the Demographic Surveillance Systems (DSS). Each of these systems has several limitations to serve as a sustained community database. If well kept, the village register can be used to collect data on births and deaths thus reducing dependency on national censuses. But the register has not gained roots in the respective villages as most of them are not frequently updated. Through the village weighing programme the under-five follow up forms have been used to collect data on immunization and nutritional status of the under fives. In spite of this, the high drop out rate of village health workers who are the kingpin to the success of the programme compromises the quality of data collected. As with the village register, the under five follow up forms faces similar problem of not being updated. Through the DSS, data on births and deaths are collected bi-annually in a selected number of districts. In some of the DSS, data on the cause of death is collected using verbal autopsies. Although the system produces reliable data on birth and death occurring at community level its use as a community database is limited by the low coverage. Currently, the system is implemented in three out of 150 districts in the country.

The RTH card provides s simple, cheap, practical and convenient method of monitoring individual child health at the under fives' clinic (Tarwa and Villiers, 2007). For a number of decades, the card has served as a tool for monitoring nutrition and vaccination status of under fives (Mukanga \& Kiguli, 2006). As home retained cards, they are reported to be well looked after by caretakers who can move with them to different places. The card provides an easy and quick reference to clinicians about the child's previous encounters with health professionals (McMaster et al., 1996). Data recorded in the card include date and type of vaccination given, and diagnosis, treatment and advice provided for each disease event. These records can be easily retrieved for community planning and in the retrospective studies (Kumar, 1993; Hagelin et al., 1991). The fact that 
current disease episodes are recorded by trained health workers makes the records on diagnoses more reliable compared to that in the DSS. Reliance on recall in establishing occurrence of a disease episode undermines reliability of the data (Cutts et al., 1990).

RTH cards have been reported to be not utilized to full potential. RTH cards can serve as a mobile databank for curative, preventive and promotive services (Tarwa and Villiers, 2007). In order for the RTH cards to serve as a sustained community database, the majority of caretakers need to retain them for at least five years. This study was conducted to determine the magnitude of RTH card possession and retention by caretakers of under fives with a view to explore the extent to which RTH cards can serve as a sustainable database for monitoring health status and interventions targeting $<5$ year children.

\section{Materials and Methods}

\section{Study site and sample selection}

This study was conducted in December 2001 and January 2002 in Korogwe town and its suburbs $\left(5^{0} 10^{\prime} \mathrm{S}, 38^{\circ} 00 \mathrm{E}\right)$ in Tanzania. With a population of 260,200 the district is estimated to have about 39,000 under fives (2002 National Population and Housing Census) with an area covering about 3700 square kilometers.

This cross-sectional study was conducted in six randomly selected wards from Korogwe town to represent urban settings and four neighboring villages to represent sub-urban settings. All children $<5$ years old found in the selected wards / villages during the study period were included in the study.

\section{Data collection}

A house to house visit was made to identify households with under-fives with the support from village and hamlet leaders who were familiar with the members of households. The caretaker (defined in this study as parent or guardian) found at home at the time of the study was interviewed using a structured questionnaire. The caretaker was asked on the number of under fives in the household and asked to produce their clinic cards (RTH cards), if they had. Information was collected on the presence of RTH card and the date of birth was recorded from the RTH card. For those who did not have a card the dates of birth was obtained from the caretakers. A calendar of local events was prepared prior to data collection and used by data collectors to facilitate recall from parents/guardians.

\section{Data analysis}

Data analysis was done using EPI Info version 6 and p-value was taken to be significant when the value was below 0.05 . Possession of RTH card was defined as the proportion of all under fives (overall) having RTH cards at the time of the study. Retention of the card was defined as the difference in card possession between the last-born under fives and the second-from-last born; and third-from-last born under fives.

\section{Research clearance}

Research clearance was obtained from the Muhimbili University College of Health Sciences. Approval for the research was obtained from the regional and district authorities. Verbal informed consent was obtained from the caretakers prior the interviews.

\section{Results}

A total of 4899 households were involved in the study in which 6364 under fives were found. Majority of the respondents were female (96.0\%). Overall, $74.3 \%$ of the underfives had RTH cards (Table 1). Possession of RTH card was found to decrease with the decrease in birth rank. Whereas, $78.3 \%$ of the last-born under fives were found to have the cards less than half of the third-from-last born under fives (45.1\%) had the cards.

Table 1: Number of respondents and the RTH card holding rate for $<5$ year by birth rank

\begin{tabular}{llllll}
\hline Birth rank & No. with cards & Percent & No. without cards & Percent & Total \\
\hline Last-born & 3834 & 78.3 & 1065 & 21.7 & 4899 \\
Second-from-last born & 822 & 63.1 & 481 & 36.9 & 1303 \\
Third-from-last born & 73 & 45.1 & 89 & 54.9 & 162 \\
Total & 4729 & & 1094 & 1635 & 6364 \\
\hline
\end{tabular}

$\chi^{2}=198.64 \mathrm{df}=2 \mathrm{p}$ value $=0.00001$ 
Analysis was done to determine some of the factors associated with possession of RTH cards. Households in which couples were married or cohabiting had higher card possession rate than those living single $(P$-value $<0.05)$. Households in which the caretaker reported to have attained primary level education had higher card possession rate $(79.7 \%)$ compared to those not educated $(74.6 \%)(P$-value $<0.05)$. Housewives were found to have higher card possession rate compared to other occupation groups (Table 2). complete vaccination schedule at the age of about one year except where child growth monitoring activities are conducted at the community level. Therefore, strategies to increase card retention need to start with enhancing the use of the cards beyond monitoring weight and vaccination at individual level. Several strategies are proposed in this paper aimed at increasing card retention by caretakers.

With supportfrom theUNICEF, quarterly

Table 2: RTH card holding rate for underfives by caretaker's marital status, education and occupation

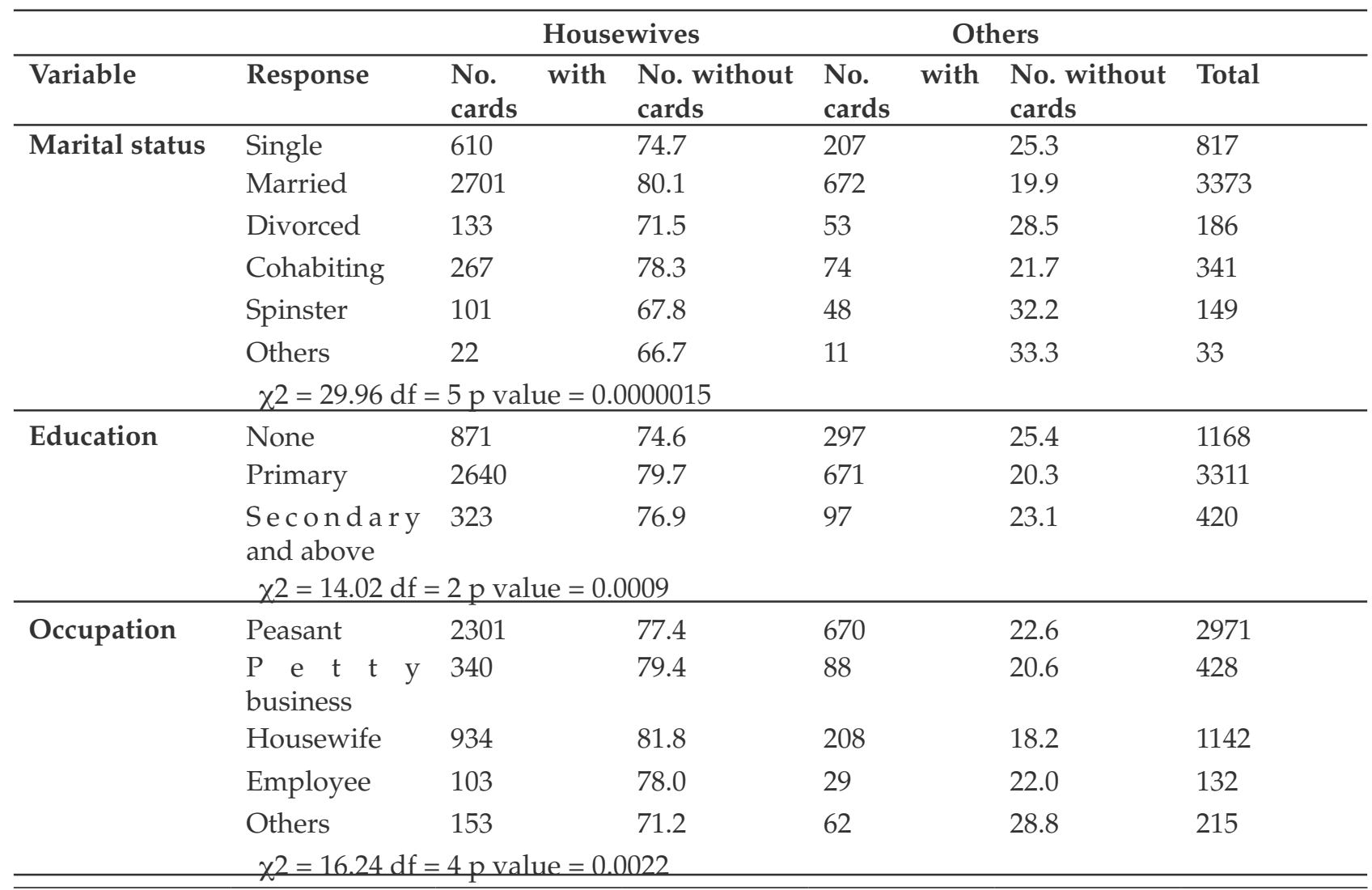

\section{Discussion}

A high rate of possession of RTH cards was found, which was similar to reports from other low income countries (Dammann \& Solarsh, 1992; Nathoo et al., 1999). The high possession rate provides an opportunity for the cards to be used to monitor and evaluate under fives health status and intervention programmes, especially in low income countries where CBHIS are inadequate or non-existent. A low rate of retention of the cards was also found, which pose a serious limitation if the cards are to serve as an effective and reliable community database. Currently, the RTH cards are mainly used for monitoring growth and vaccination status of individual child. Many caretakers do not usually take children to the clinics once they village health days are conducted by the village governments under the Child Survival, Protection and Development programme(CSPD) in $40 \%$ of Tanzanian villages (Johnson, 2005). One of the main activities during these days is to follow-up health status of under fives using RTH cards. There is need to roll-out these activities throughout the country and strengthening them where they already have started. Members of the village government need to be trained on evaluation of health interventions in their respective communities using simple indicators. They should be supported to collect and aggregate only the data that could help them to monitor and perform their activities better (Husein et al., 1993) and take the necessary action for the identified problems.

In many low income countries, a large 
proportion of people do not seek for modern care when they get sick. Consequently, it is not possible to adequately measure outcome and impact of intervention programmes using health facility data. If trained and supported, health workers can use data collected in the community to complement facility based data thus obtain a comprehensive picture of their catchment areas. Using RTH cards as the database, district health authorities can conduct baseline and subsequent surveys to evaluate outcome and impact of interventions targeting under fives in their respective districts (Bachmann \& Barron, 1996). Detailed analyses of community based data collected at health facility level can be done at district level where better analytical skills and computerized systems exists.

Studies have shown that despite being an overburden to health workers as a result of collecting enormous amount of data, facility based Health Information System (HIS) is often unreliable (Robey \& Lee, 1990; Simba \& Mwangu, 2005). Collection of outcome and impact indicator data at the community level will reduce the need to collect same data in the health facilities. This will allow de-bulking of the facility based HIS thus reducing the burden of data collection hence improving data quality.

Strategies to enhance retention of RTH cards after the completion of vaccination schedule might include: (i) Using the RTH cards as a criterion for under fives to qualify for services earmarked for the age group. These might include exemption to user-fees when seeking medical care and to procure insecticide treated nets at a subsidized price through the current voucher scheme. In many areas, the card is often the only evidence to determine the actual age of the child; and (ii) Giving special attention to caretakers who come with RTH cards at the health facilities, for example, giving them first priority to be attended.

Having a permanent or semi permanent RTH book (passport) to cater for promotional, preventive as well as curative services will motivate caretakers to retain it for future use, especially if a token fee is charged (Van der Hoek, 1994). The book can be used by clinicians to record briefly the disease suffered, treatments provided and if referral advice was given. In addition to being useful in the follow up of individual child health care through the referral ladder the notes can also serve as a database for future research. Despite some limitations in recording disease data (Tarwa \& Villiers, 2007) other studies have shown such a book to be acceptable by the majority of the health workers and clients (Harrison et al., 2005).

Selection of Korogwe township was based on convenience. This might limit generalization of findings from this study. The fact that the study area comprised of an urban and sub- urban areas might lead to overestimation of proportion of card possession and retention since service coverage tend to be better in urban compared to rural areas.

In conclusion, the high rate of possession of RTH card among under fives creates an opportunity for the cards to serve as a community database. If retained in high proportion, the cards can be useful to monitor health status of under fives and evaluate intervention programmes targeting them. Strategies are needed to maintain a high proportion of card possession and increase card retention.

Received 18 November 2008

Revised 28 November 2008

Accepted 2 December 2008

\section{References}

Bachmann, M.O. \& Barron, P. (1996) Missed opportunities for immunization in curative and preventive services in a community health centre. A follow up survey. South African Medical Journal 86, 947-949.

Cutts, F.T., Smith, P.G. \& Colombo, S. (1990) Field evaluation of measles vaccine efficacy in Mozambique. American Journal of Epidemiology 131, 349-355.

Dammann, D.F. \& Solarsh, G.C. (1992) The use of COSAS in the analysis of vaccination coverage in urban, peri-urban and rural populations. South African Medical Journal 82, 118-123.

Hagelin, E., Lagerberg, D. \& Sundelin, C. (1991) Child health records as a database for clinical practice, research and community planning. Journal of Advanced Nursing 16, 15-23.

Harrison, D., Harker, H., Heese, H. \& Mann, M. (2005) An assessment by nurses and mothers of a 'Road to Health' Book in the Western Cape. Curationis 28, 57-64.

Husein, K., Adeyi, O., Bryant, J. \& Cara, N.B. (1993) Developing a primary health 
care management information system that supports the pursuit of equity, effectiveness and affordability. Social Science and Medicine 36, 585-596.

Johnson, U. (2005) Human Rights Approach to Development Programming. UNICEF. Nairobi. Kenya. Pg 111.

Kumar, R. (1993) Streamlined records benefit maternal and child health care. World Health Forum 14, 305-307.

McMaster, P. McMaster, H. \& Sothall, D. (1996) Personal child health record and advice booklet programme in Tuzia, Bosnia Herzegovina. Journal of the Royal Society of Medicine 89, 202-204.

Mukanga, D. \& Kiguli, S. (2006) Factors affecting the retention and use of child health cards in a slum community in Kampala, Uganda, 2005. Maternal Child Health 10, 545-552.

Nathoo, K.J., Bannerman, C.H. \& Pirie, D.J. (1999) Pattern of admissions to the paediatric medical wards (1995 to 1996) at Harare Hospital, Zimbabwe Central
African Journal of Medicine 45, 258-263.

Robey, J.M. \& Lee, S.H. (1990) Information system development in support of national health programme monitoring and evaluation: The case of Philippines. World Health Statistics Quarterly 43, 3743.

Simba, D.O. \& Mwangu, M.A. (2005) Quality of a routine data collection system for health: case of Kinondoni district in the Dar es Salaam region, Tanzania. South African Journal of Information Management 7 (2). Available at: http://www.sajim.co.za]

Tarwa, C. \& Villiers, P. (2007) The use of the road to health card in monitoring child health. South African Family Practice 49 (1). Available at: http://www. safpj.co.za/index.php/safpj/article/ viewFile/486/637]

Van der Hoek, W., Ngoma, M., Soeters, R. (1994) The home-based health passport: a tool for primary health care. Tropical Doctor 24, 21-23. 